\title{
Urgences
}

\section{Octobre : jardin figé}

\section{Louise Cotnoir et Hugues Corriveau}

Numéro 16, mars 1987

D.G. Jones : d'un texte, d'autres

URI : https://id.erudit.org/iderudit/025377ar

DOI : https://doi.org/10.7202/025377ar

Aller au sommaire du numéro

Éditeur(s)

Urgences

ISSN

0226-9554 (imprimé)

1927-3924 (numérique)

Découvrir la revue

Citer ce document

Cotnoir, L. \& Corriveau, H. (1987). Octobre : jardin figé. Urgences, (16), 28-29.

https://doi.org/10.7202/025377ar d'utilisation que vous pouvez consulter en ligne.

https://apropos.erudit.org/fr/usagers/politique-dutilisation/ 


\section{Louise Cotnoir/Hugues Corriveau OCTOBRE: JARDIN FIGÉ}

Sous l'averse, il y a la ruine

des ombres, il y a

le tombeau des fleurs

S'y cueillent les couleurs

des feuilles tombées. Figées

il y a les pierres qui fleurissent

comme un recueil de fragments

où ceux-là qui ne sont plus continuent

à faire signe

De plus en plus ma bouche

est pleine de pierres

et les restes de mes semblables

rappellent ces fleurs

Y a-t-il là, confusion, illusion

ou Angkor Wat

ou le coeur de la cité après

dix heures le soir? Il n'y a là

ni vie ni mort

ni humain. J'y songe

sous l'averse, sombrement. Il y a là

une résurgence des runes.

Les choix que nous avons décidé de respecter:

a) Nous avons privilégié l'expression française «il y a» plutôt que le "c'est» pour traduire «it is», d'abord parce qu'il nous paraissait que l'effet de répétition de la première expression était plus fort, plus "perceptible" dans la traduction, ensuite parce qu'elle nous permettait de réutiliser certains éléments qui la constituent, comme Jones luimême le fait en anglais: par exemple, nous avons pu, dans les vers 4 et 19 , reprendre le "y» dans «S'y cueillent" et «J'y songe» comme Jones utilisait, lui, le «it» dans «It gathers» et «l pass it».

b) Nous avons respecté, dans la mesure du possible en français, la position même des mots, tels qu'ils apparaissent dans le texte anglais; 
comme nous avons absolument respecté les vers eux-mêmes dans chacun de leurs constituants.

c) De plus, nous avons décidé de privilégier la répétition lexicale comme dans le texte anglais plutôt que la diversité du vocabulaire, de telle sorte que nous répétons, nous aussi, les mots que Jones luimême reprend dans le texte original: ainsi en est-il des mots «fleurs» (v. 3 , v. 13) et «fleurissent» (v. 6) correspondant aux "flower(s)" anglais; «averse» (v. 1, v. 20) correspondant au mot «rain»; «pierres» (v 6., v. 11) correspondant au mot "stones" (ce parti pris explique pourquoi nous n'avons pas traduit le vers 11 «is full of stones» comme nous aurions été tentés de le faire par «se pétrifie» ou par «se scelle», car il nous apparaissait plus important de donner deux fois le mot «pierres» comme Jones le fait pour «stones»).

d) Nous avons de plus tenté, là encore dans la mesure du possible, de reproduire certaines assonances afin de rendre quelques fois le travail phonétique du texte anglais; ainsi en est-il des mots «cueillent» (v. 4) et «recueil» (v. 7) qui reproduisent exactement, et à la même place stratégique qu'ils occupent dans chacun de leur vers respectif, les mots "gathers» et "gathering» du texte d'origine. C'est également la même raison qui nous a fait garder les mots «ruine» (v. 1, «ruin») et «runes» (v. 21, «runes») afin d'en conserver les valeurs sémantiques et phonétiques plutôt que de chercher à traduire, par exemple, le palimpseste présent dans le mot "rune» ou le désastre dans le mot «ruine».

e) Il serait intéressant de noter que nos partis pris redonnent au texte français une spatialité sur la feuille assez semblable à celle du texte anglais.

f) La difficulté de traduire ce texte vient peut-être du fait qu'il s'apparenterait à la forme du ukenning» que Thierry Haumont dans Le conservateur des ombres (Gallimard, 1984, p. 248-249) définit comme «une conjonction de métaphores. Une périphrase métaphorique; un emboîtement de ces périphrases.» De plus, il "rattache le "kenning" au vieux genre littéraire de l'énigme». Mais ce qu'il est avant tout intéressant de noter c'est que Jones fait référence aux «runes» qui se rapportent à «l'ancien alphabet des langues germaniques orientales (gothique ou gotique) et septentrionales (nordique ou norroise)». "Il faut savoir que le «kenning" appartient également à la «littérature norroise». Or, quand on sait que les «runes» sont très souvent gravées sur "pierre» (stone), ne serions-nous pas en présence justement d'un «kenning runique»? 
g) Nous aimerions faire remarquer que nous avons respecté l'orthographe du mot «Wat» tel qu'il était écrit sur le manuscrit qui nous a été remis. Signalons en passant que, s'il est vrai que le mot «Wat» peut s'écrire en français avec un "W» ou un " $V$ », nous n'avons jamais trouvé, dans les dictionnaires dont nous disposions, le nom d' «Angkor Vat» écrit avec un «W» mais toujours avec « $V »$.

\section{Jean-Paul Daoust/Mario Savoie JARDIN DE PIERRES: OCTOBRE}

Dans la pluie, c'est une ruine hantée, c'est

un tombeau fleuri

Qui ramasse les couleurs

des feuilles mortes. Pourtant

ses pierres fleurissent

comme un fouillis de livres

où les fantômes continuent de

parler

De plus en plus ma bouche

est pleine de pierres

et les os de mes semblables

ressemblent aux fleurs

N'est-ce qu'un enchevêtrement, un paradis

ou Angkor Vat

ou le centre-ville passé

10:00 P.M.? Ce n'est

ni mort ni vivant

ni humain. Je le longe

dans la pluie sombrement. C'est

la naissance des écrits secrets 